\title{
The CMS Level-1 Tau algorithm for the LHC Run II
}

\section{Luca Cadamuro* on behalf of the CMS collaboration}

LLR - Ecole Polytechnique and CNRS

E-mail: luca.cadamuro@cern.ch

The CMS experiment relies on a two-level online selection system that achieves a rejection factor of nearly $10^{5}$. The first level (L1) is based on coarse information coming from the calorimeters and the muon detectors while the High Level Trigger combines fine-grain information from all subdetectors. During Run II, the centre-of-mass energy of the LHC collisions will be increased to 13 or $14 \mathrm{TeV}$, and progressively an instantaneous luminosity of $10^{34} \mathrm{~cm}^{-2} \mathrm{~s}^{-1}$ will be reached. To guarantee a successful and ambitious physics program in this intense environment, the CMS Trigger and Data Acquisition system must be upgraded. The L1 Calorimeter Trigger hardware and architecture will be upgraded in particular, allowing sophisticated algorithms to be deployed. These algorithms will better exploit the calorimeter granularity and will open the possibility of making correlations between different parts of the detector. In this context, an optimised tau algorithm, implementing an innovative dynamic clustering technique, has been developed for the selection of hadronically decaying tau leptons, which represents a real challenge for an electronics trigger system. The performance of this tau trigger will be demonstrated, both in terms of efficiency and rate reduction. The different handles to control trigger rates in different pile-up scenarios will be described.

The European Physical Society Conference on High Energy Physics 22-29 July 2015

Vienna, Austria

\footnotetext{
* Speaker.
} 


\section{Introduction}

During the Run II the Large Hadron Collider (LHC) will deliver proton-proton collisions under different conditions than the Run I. In particular, the center-of-mass energy will be increased from 8 to $13 \mathrm{TeV}$ with an instantaneous luminosity of $10^{34} \mathrm{~cm}^{-2} \mathrm{~s}^{-1}$, and on average 40 collisions per bunch crossing (denoted as pile-up or PU in the following).

The intense pile-up conditions are particularly challenging for the trigger system of the CMS experiment. In particular, the current Run I Level-1 (L1) calorimeter trigger of the CMS detector will not be able to provide an efficient identification of hadronic decays of $\tau$ leptons at a sustainable trigger rate.

For this reason, the L1 calorimeter trigger system will be entirely upgraded. It will use a new hardware architecture based on the MicroTCA technology. The system is being installed and it will be operational starting from the 2016 LHC runs.

\section{The CMS detector}

The CMS detector is composed of several concentric subsystems that are devoted to the detection of specific particle interactions. The L1 calorimeter trigger is based on the information detected in the Electromagnetic Calorimeter (ECAL) and Hadron Calorimeter (HCAL) subdetectors. This information is organized into trigger towers (TT), regions of the calorimeters extending approximately 0.087 both in the $\eta$ and $\phi$ directions over most of the detector, with some differences in the endcap region. The TT are the basic objects used by the L1 calorimeter trigger algorithms.

More details about the L1 2016 upgrade calorimeter trigger can be found in [1].

\section{Algorithm description}

The L1 $\tau$ candidates are created by regrouping TT into clusters. Properties such as isolation and energy are computed in subsequent steps to provide a good description of the $\tau$ hadronic decay and an efficient background rejection.

Four main steps of the algorithm can be defined: clustering and merging, calibration, isolation, and shape veto.

\section{Clustering and merging}

Clusters are built by dynamically regrouping the TT according to their energy, summing ECAL and HCAL information. Transverse energies, i.e. the projection of the momentum vectors pointing to the calorimeter cells in the direction orthogonal to the beam axis, are used. In the following, the term "energy" and the symbol $E_{T}$ will denote the transverse energy.

Cluster creation is seeded by local maxima of energy above a fixed threshold. Only the TT giving the most significant contribution to the cluster energy are regrouped together in order to get a good description of the $\tau$ decay while minimizing contributions from other sources such as the pile-up. More details about the clustering technique can be found in [3].

The charged particle trajectories from $\tau$ decays are bent by the magnetic field of the CMS detector. For this reason, the energy deposits of neutral and charged $\tau$ decay products, as well as 
those of oppositely charged particles, will be spatially separated inside the calorimeter, especially along the $\varphi$ direction. In order to take this effect into account and to improve the reconstruction of such decays, nearby clusters can be merged into a single L1 $\tau$ candidate. A requirement on the relative position of the two clusters seed is applied in order to perform the merging.

\section{Calibration}

The energy of the L1 $\tau$ candidate is computed from the sum of the energies of the clustered TT. Calibration is performed using separately the ECAL and HCAL energies, that are combined using the following function:

$$
\begin{array}{ll}
E_{T}^{\tau}=\left(a E_{T}^{E C A L}+b E_{T}^{H C A L}+p\right) \cdot d(\eta) & \text { if } \quad E_{T}^{E C A L}>0 \\
E_{T}^{\tau}=\left(c E_{T}^{H C A L}+q\right) \cdot d(\eta) & \text { if } \quad E_{T}^{E C A L}=0
\end{array}
$$

where $E_{T}^{\tau}, E_{T}^{E C A L}$ and $E_{T}^{H C A L}$ denote respectively the calibrated $\tau$ energy and the sum of the ECAL and HCAL energies of the clustered TT. The values of $a, b$ and $c$ depend on the uncalibrated energy $E_{T}^{\text {quncal }}=E_{T}^{E C A L}+E_{T}^{H C A L}$ and on the barrel/endcap position of the cluster (one value for each detector region). The constants $p$ and $q$ also depend on the barrel/endcap position, while the $d$ coefficient depends on the value of the coordinate $\eta$ of the cluster.

\subsection{Isolation}

Isolation energy is derived from the sum of the TT energies inside a window of size $5 \times 9$ in the $(\eta, \varphi)$ plane around the $\mathrm{L} 1$ candidate. This value is denoted as $E_{T}^{5 x 9}$. Isolation energy $E_{T}^{i s o}$ is therefore computed as:

$$
E_{T}^{\text {iso }}=E_{T}^{5 \times 9}-E_{T}^{\tau \text { uncal }}
$$

which is non-negative by construction.

Isolation of the L1 $\tau$ candidate is determined by comparing $E_{T}^{i s o}$ to a threshold $\lambda$, the value of which depends on the $\eta$ position, on $E_{T}^{\tau \text { uncal }}$ itself and on the amount of pile-up in the event. The latter is estimated at the hardware level as the number of TT with $E_{T}>0$ in the 8 central $\varphi$ "rings" of the detector. In this way pile-up effects are taken into account: the stability of the isolation versus the PU is shown in figure 1; the distribution is approximately flat over all the PU range expected for Run II, with an efficiency loss smaller than 5\% at high PU.

Isolation thresholds have been designed in order to have a constant efficiency over the whole detector for all values of PU and $\tau$ energies.

\subsection{Shape veto}

The shape of the L1 $\tau$ candidate resulting from the clustering phase is exploited for additional background rejection. Possible cluster shapes are ordered according to the $S / B$ ratio, with $S(B)$ denoting the probability for a signal (background) process to produce such a shape. Clusters with a shape with low $S / B$ are not considered as valid L1 $\tau$ candidates, allowing one to reject some background independently from the isolation requirement. Of course, such a rejection has an impact also on signal efficiency. In the following results, a threshold corresponding to a $97 \%$ signal efficiency and $10 \%$ background rejection is used. 


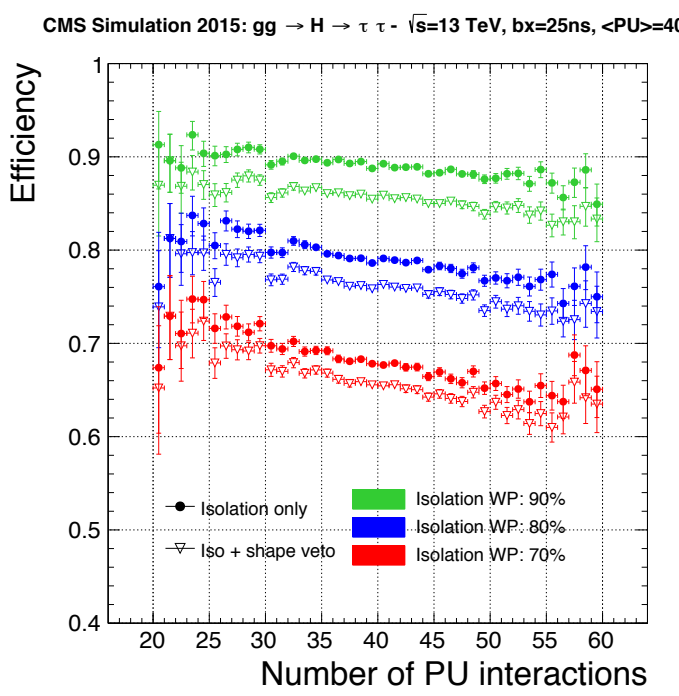

Figure 1: $\tau$ trigger efficiency in presence of isolation versus the number of pile-up interactions in the event.

\section{Performance}

Performance is evaluated using a simulation of the CMS detector. The simulated signal process is the production of a SM Higgs boson by gluon fusion with a subsequent decay into a $\tau$ pair. The simulated background is a pile-up of multiple collisions that brings hadronic activity in the detector. The performance of the $\mathrm{L} 1$ algorithm is evaluated with respect to hadronic $\tau$ decays reconstructed offline using the particle flow based technique. These are required to have $p_{T}>20 \mathrm{GeV}$ and $|\eta|<2.1$ and be geometrically matched with a generated hadronic $\tau$ decay. The performance of the 2016 upgrade algorithm is compared to that of the Run I algorithm.

Spatial resolution ( $\eta$ position) and energy resolution are shown in figure 2 . The improvement in the spatial resolution of the upgrade algorithm is clearly visible, and this is due to the improved granularity of the new system. Also, the energy resolutions are comparable for both algorithms and this result is achieved with the upgrade algorithm by using only about $10 \%$ of the TT with respect to the Run I algorithm. Energies of the Run I algorithm candidates have been rescaled here and in all following plots by a factor 0.628 in order to center the mean of the distribution to 1 for a more direct comparison.

The trigger efficiency as a function of $p_{T}$ is shown in figure 3(a). The black line is computed for the upgrade trigger in absence of isolation, and the green, blue and red lines correspond to the 90\%, 80\% and 70\% isolation efficiency working points (WP). The dashed lines correspond to the additional application of the shape veto. The orange line denotes the Run I algorithm.

An energy threshold on the $\mathrm{L} 1 \tau$ candidates of $30 \mathrm{GeV}$ is applied in all cases; the slope of the curve around $30 \mathrm{GeV}$ is therefore related to the energy resolution and to the change of isolation efficiency as a function of $p_{T}$. The 2016 upgrade algorithm achieves a flat plateau efficiency even after the isolation requirement, while the Run I algorithm is affected by an efficiency drop at high $p_{T}$. The efficiency is significantly lower over the whole $p_{T}$ range for the Run I algorithm.

The expected rate for the di- $\tau$ trigger for collision conditions of Run II ( $25 \mathrm{~ns}$ bunch spacing 


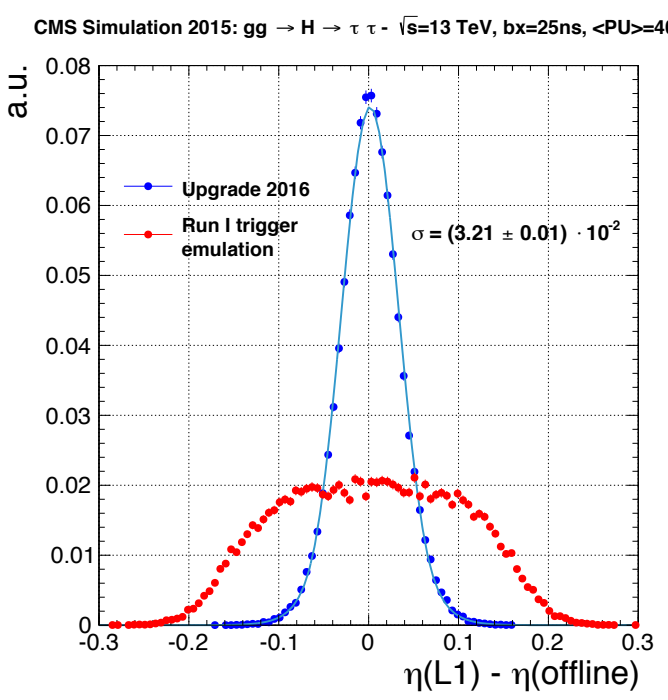

(a)

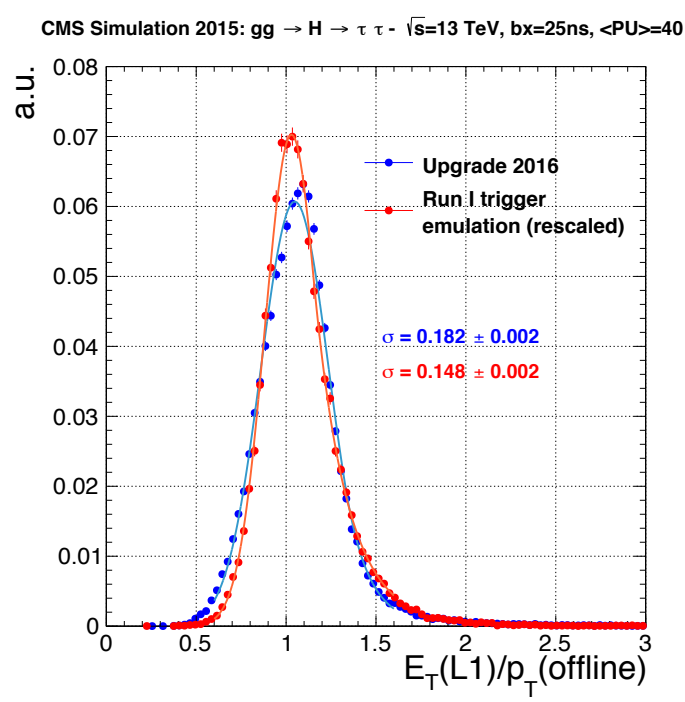

(b)

Figure 2: Spatial resolution of the $\eta$ coordinate (a) and energy resolution (b) for the upgrade trigger (blue) and Run I trigger (red). The fit functions are a Crystal Ball function (a) and a double Crystal Ball (b). The value of the $\sigma$ of the gaussian core from the fit is reported on the plot.

and $40 \mathrm{PU}$ on average) is shown in figure 3(b). The rate is shown as function of the threshold applied on both L1 $\tau$ candidates. The target rate is $3 \mathrm{kHZ}$, which corresponds to the available bandwidth for this trigger. For this rate value, the L1 thresholds for the upgrade algorithm range between 30 (29) and 42 (40) GeV depending on the WP of the isolation without (with) shape veto. The threshold for Run I is $32 \mathrm{GeV}$.

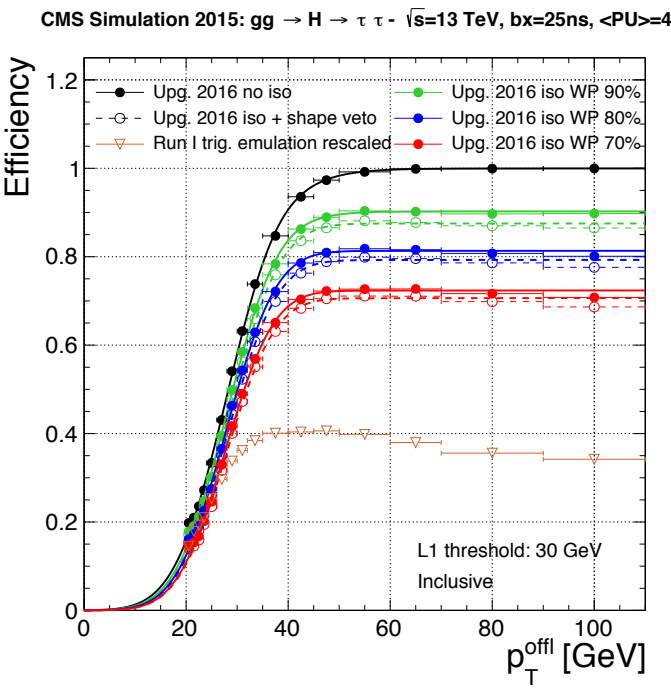

(a) Turn-on with $\mathrm{L} 1 p_{T}>30 \mathrm{GeV}$

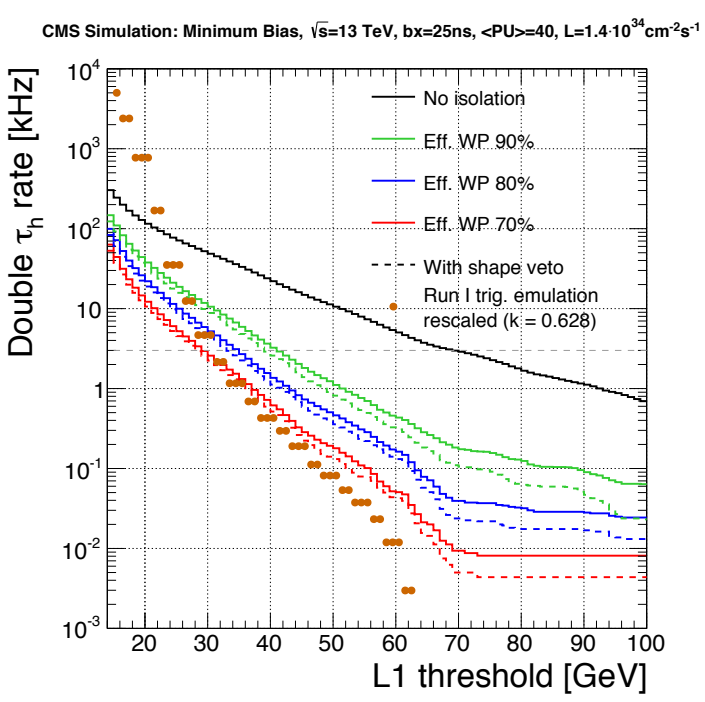

(b) Rate for di- $\tau$ trigger

Figure 3: Efficiency as a function of $p_{T}$ (a) and di- $\tau$ rate as a function of the $\mathrm{L} 1 p_{T}$ threshold (b).

The trigger efficiency on signal versus the background rejection is shown in figure 4 . The 
performance of the upgrade algorithm is significantly better than the Run I system, as for the same background rejection (i.e. rate) the signal efficiency is significantly higher. In particular, the isolation requirement results in an efficient background control and rejection.

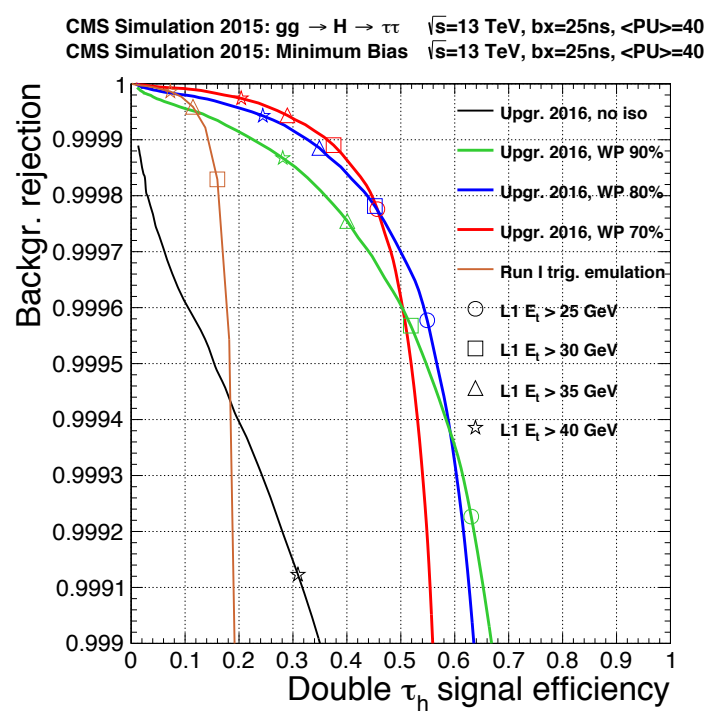

Figure 4: Efficiency of double $\tau$ trigger on $g g \rightarrow H \rightarrow \tau \tau$ versus background rejection. Each curve is computed by varying the value of the L1 threshold applied on both L1 $\tau$ candidates.

\section{Conclusions}

The reconstruction of $\tau$ hadronic decays is an extremely challenging task for a hardware system, especially at a hadron collider and with high levels of pile-up. Nevertheless, the 2016 upgrade $\tau$ algorithm achieves a very good performance both in terms of efficiency and rate control, significantly improving the CMS triggering capabilities with respect to the Run I system.

The new system will have a large, positive impact on all the analyses involving $\tau$ leptons in the final state, such as the study of the Higgs boson decay into $\tau$ lepton pairs.

\section{Acknowledgment}

The work of the author of this paper has been partly funded by the P2IO LabEx (ANR-10LABX-0038) in the framework "Investissements d'Avenir" (ANR-11-IDEX-0003-01) managed by the French National Research Agency (ANR).

\section{References}

[1] CMS Collaboration, CMS technical design report for the level-1 trigger upgrade, CERNLHCC-2013-011, CMS-TDR-12 (2013)

[2] CMS Collaboration, L1 calorimeter trigger upgrade: tau performance, CMS DP-2015/009 (2015) 
[3] J. B. Sauvan for the CMS Collaboration, Performance and upgrade of the CMS electron and photon trigger for Run 2, J. Phys. Conf. Ser. 587 (2015) 1, 012021. 